\title{
Repercussões da sífilis na gestação: Possibilidades de atuação da enfermagem
}

\author{
Repercussion of sphilis during pregnancy: Possibilities of nursing performance \\ Efectos de la sífilis sobre el embarazo: Possibilidades de acción em enfermería
}

\author{
Emilie dos Santos Aleluia \\ ORCID: https://orcid.org/0000-0002-7231-2682 \\ Centro Universitário Jorge Amado, Brasil \\ E-mail:emilie.aleluia@gmail.com \\ Luana dos Reis Nascimento \\ ORCID: https://orcid.org/0000-0001-8378-2586 \\ Centro Universitário Jorge Amado, Brasil \\ E-mail: luareis@gmail.com \\ Vitoria Vilas Boas da Silva Bomfim \\ ORCID: https://orcid.org/0000-0003-4897-0279 \\ Centro Universitário Jorge Amado, Brasil \\ E-mail: pesquisaclinica9@gmail.com \\ Andreia Silva Rodrigues \\ ORCID: https://orcid.org/0000-0002-0091-2849 \\ Centro Universitário Jorge Amado, Brasil \\ E-mail: enfandreiarodrigues@ @otmail.com \\ Maria Dhescyca Ingrid Silva Arruda \\ ORCID: https://orcid.org/0000-0002-9073-7844 \\ Faculdade São Francisco da Paraíba, Brasil \\ E-mail: dhescycaingrid20@gmail.com \\ Angelica Ribeiro do Nascimento Oliveira \\ ORCID: https://orcid.org/0000-0002-7240-2004 \\ Centro Universitário Maurício de Nassau, Brasil \\ E-mail: angelicalribeiro.ar19@gmail.com \\ Maria Eduarda Wanderley de Barros Silva \\ ORCID: https://orcid.org/0000-0002-4642-3282 \\ Universidade Federal de Campina Grande, Brasil \\ E-mail: eduarda.wanderly@ outlook.com \\ Sabrina Iracema da Silva Couto \\ ORCID: https://orcid.org/0000-0002-3864-4843 \\ Uninassau Caruaru, Brasil \\ E-mail: sabrinacouto89@gmail.com \\ Thaiz Helena Vicente Ramos \\ ORCID: https://orcid.org/0000-0003-0501-261X \\ Universidade Castelo Branco, Brasil \\ E-mail: thaizgtg@gmail.com \\ Daniela de Oliveira Holanda \\ ORCID: https://orcid.org/0000-0003-3495-6003 \\ Faculdade de Palmas, Brasil \\ E-mail: danielaholandaenfermagens@gmail.com \\ David Matias Souza \\ ORCID: https://orcid.org/0000-0001-9408-0467 \\ Universidade Castelo Branco, Brasil \\ E-mail: davidmatiassouza123@gmail.com
}

\begin{abstract}
Resumo
Objetivo: conhecer as repercussões da sífilis na gestação e a possibilidade de atuação da enfermagem. Metodologia: Revisão integrativa da literatura realizada em julho de 2020 na BVS, SciELO e PubMed, utilizando-se os DeCS: assistência de enfermagem, sífilis, saúde materno-infantil, pré-natal e gestação. Selecionaram-se artigos originais na íntegra, em português, inglês e espanhol, publicados entre 2015 a 2020. Resultados: Os 3 artigos selecionados abordaram as temáticas: repercussões da sífilis na mãe; consequências da transmissão vertical para o feto e/ou neonato e possibilidades de atuação da enfermagem. As principais repercussões da sífilis para a mãe foram danos psicológicos, e para o neonato, o aparecimento precoce ou tardio da infecção podem resultar em sequelas a curto ou longo prazo. Frente a isso, a enfermagem deve atuar durante todo processo assistencial, em ações educativas como promoção, prevenção, diagnóstico precoce e tratamento. Considerações Finais: A sífilis traz sérias repercussões para o binômio materno fetal e diante disto a enfermagem tem papel fundamental no combate à este patógeno, buscando frear o mais abruptamente rápido os possíveis danos causados à saúde materno-infantil.
\end{abstract}


Palavras-chave: Assistência de enfermagem; Sífilis; Saúde materno-infantil; Pré-natal; Gestação.

\begin{abstract}
Objective: to know the repercussions of syphilis on pregnancy and the possibility of nursing work. Methodology: Integrative literature review carried out in July 2020 in the VHL, SciELO and PubMed, using the DeCS: nursing care, syphilis, maternal and child health, prenatal care and pregnancy. Original articles in full, in Portuguese, English and Spanish, published between 2015 and 2020, were selected. Results: The 3 selected articles addressed the themes: impact of syphilis on the mother; consequences of vertical transmission for the fetus and/or neonate and possibilities for nursing work. The main repercussions of syphilis for the mother were psychological damage, and for the newborn, the early or late appearance of the infection can result in short or long term sequelae. Faced with this, nursing must act throughout the care process, in educational actions such as promotion, prevention, early diagnosis and treatment. Final Considerations: Syphilis has serious repercussions for the maternal-fetal binomial and, in view of this, nursing has a fundamental role in combating this pathogen, seeking to curb the possible damage caused to maternal and child health as quickly as possible.
\end{abstract}

Keywords: Nursing care; Syphilis; Maternal and child health; Prenatal care; Pregnancy.

\begin{abstract}
Resumen
Objetivo: conocer las repercusiones de la sífilis en el embarazo y la posibilidad del trabajo de enfermería. Metodología: Revisión integrativa de la literatura realizada en julio de 2020 en la BVS, SciELO y PubMed, utilizando el DeCS: atención de enfermería, sífilis, salud maternoinfantil, atención prenatal y embarazo. Se seleccionaron artículos originales completos, en portugués, inglés y español, publicados entre 2015 y 2020. Resultados: Los 3 artículos seleccionados abordaron los temas: impacto de la sífilis en la madre; consecuencias de la transmisión vertical para el feto y / o neonato y posibilidades de trabajo de enfermería. Las principales repercusiones de la sífilis para la madre fueron el daño psicológico, y para el recién nacido, la aparición precoz o tardía de la infección puede resultar en secuelas a corto o largo plazo. Ante esto, la enfermería debe actuar a lo largo del proceso asistencial, en acciones educativas como la promoción, la prevención, el diagnóstico precoz y el tratamiento. Consideraciones Finales: La sífilis tiene graves repercusiones para el binomio materno-fetal y, por ello, la enfermería tiene un papel fundamental en el combate de este patógeno, buscando frenar lo más rápidamente posible los posibles daños ocasionados a la salud maternoinfantil.
\end{abstract}

Palabras clave: Atención de enfermería; Sífilis; Salud maternoinfantil; Atención prenatal; Embarazo.

\title{
1. Introdução
}

As infecções sexualmente transmissíveis (IST) correspondem a uma das patologias transmissíveis mais comuns, afetando a saúde e a vida dos indivíduos mundialmente, consideradas como problema de saúde pública, por acarretar impactos diretamente na saúde reprodutiva e infantil, levando a repercussões a curto e longo prazo. No Brasil os números de casos de sífilis, entre homens e mulheres, são alarmantes e precisa ser controlado (BRASIL, 2019).

A sífilis correlaciona a um quadro patológico infeccioso provocado pela bactéria espiroqueta Treponema pallidum, que transigi transmissões por via sexual, transfusão sanguínea ou com objetos contaminados, podendo ocorrer surtos e períodos de latência de duração variável. Em período gestacional representa uma série de alterações clínicas, podendo ocasionar aborto, morte perinatal, prematuridade ou sífilis congênita (BRASIL, 2012; Damasceno, Monteiro, Rodrigues, Barmpas, Cerqueira \& Trajano, 2014).

A transmissão de infecções durante a gestação pode ocorrer por transmissão hematogênica placentária, sucede durante a passagem pelo canal de parto, ou por transmissão vertical intra uterina através do sangue do cordão umbilical. As infecções congênitas são denominadas de infecções adquiridas devido a transfusão materno fetal. Inúmeros são os fatores que contribuem e designam de infecções gestacionais e refletem ao acometimento fetal, entretanto destaca-se a idade gestacional e a defesa placentária (Araújo, 2015; Guerra, da Costa, dos Santos, da Silva \& Barcelos, 2017).

Os dados Epidemiológicos comprovam uma prevalência de 2009 a 2016 de 6,3 milhões de casos de sífilis estimados pela Organização Mundial de Saúde. O boletim epidemiológico número 01 de outubro de 2019, retratou sobre sífilis no Brasil. Observou-se que a doença infecciosa sexual adquirida teve a taxa de detecção aumentada, notificados no Sistema de Informação de Agravos de Notificação (Sinan) 158.051 casos em 2018, ou seja, 75,8\% de casos, número maior que os casos 
registrados em 2017 que foi 59,1\%. Em 2018 cerca de 62,599 casos de sífilis em gestantes (taxa de 21,4\%), 26,219 casos de sífilis congênita (9\%) e 241 óbitos $(8,2 \%)$. Nota-se neste período uma elevação de casos notificados, impactando a saúde dos indivíduos e a política pública (BRASIL, 2019).

Em 1983 com o intuito de atender as necessidades e organização da atenção à saúde das mulheres diante do contexto e dos determinantes de saúde, o Ministério da Saúde criou o Programa de Assistência Integral à Saúde da Mulher (PAISM), que possibilitou ações governamentais de políticas públicas de saúde feminina. O programa visava a ampliação de políticas de atenção à saúde da mulher integralmente, com atenção humanizada, abrangendo todas as etapas, promoção, prevenção, reabilitação, planejamento familiar, reprodução, questões relativas a infecções sexualmente transmissíveis (IST), dentre outros (Andreucci \& Cecatti, 2011).

Durante a gestação a mulher passa por mudanças fisiológicas que necessitam de cuidados específicos, pois a falta de acompanhamento adequado pode acarretar sérias complicações tanto para a gestante quanto para o feto. Esses cuidados e orientações desenvolvidas ao longo do processo gestacional é denominada de pré-natal. A atenção pré-natal está enquadrado no programa Estratégia Saúde da família e respaldado pelo Programa de Humanização ao Pré-Natal e Nascimento (PHPN) que integra a Política Nacional de Saúde da Mulher (PNSM) (Andreucci \& Cecatti, 2011; Magalhães, Kawaguchi, Dias \& Paranhos Calderon, 2011).

Estudos apontam que há uma falha na educação em saúde no período pré-natal e perinatal o que favorece para a ocorrência da sífilis gestacional e congênita. Um déficit da assistência ao pré-natal pode ocasionar em falhas no diagnóstico e tratamento da gestante infectada e do seu parceiro, que precisam estar cientes sobre os riscos e complicações associados à não adesão do tratamento. Outrossim, pode-se evitar a reinfecção e novos casos na mesma mulher (BRASIL, 2017). A enfermagem exerce papel fundamental, pois tem contato direto com os pacientes realizando as consultas e orientações, testes rápidos, identificação da sintomatologia, acompanhando e oferecendo educação em saúde (BRASIL, 2015; Duarte, Borges \& de Arruda, 2011).

O(a) enfermeiro(a) lida diretamente com o binômio mãe-bebê e sua família frente a sífilis gestacional e congênita, considerando-se a complexidade diagnóstica e assistencial do agravo, com adoção de medidas que possam minimizar riscos, contribuindo para redução da morbimortalidade materno-fetal. $\mathrm{O}$ atendimento da enfermagem possibilita uma extensão na cobertura e melhora da qualidade assistencial, de forma humanizada, com condutas acolhedoras, acessível e com ações que integram todos níveis da atenção à saúde da mulher (BRAIL, 2013; de Figueiredo, Cavalcante, de Oliveira, Monteiro, da Silva Quirino \& de Oliveira, 2015).

Diante do exposto, a questão norteadora da pesquisa foi: Quais são as repercussões da sífilis na gestação? E quais possibilidades de atuação da enfermagem? A partir dessa, objetivou-se conhecer as repercussões da sífilis na gestação bem como descrever as possibilidades de atuação da enfermagem.

\section{Metodologia}

O presente estudo trata-se de uma revisão integrativa da literatura, a partir de levantamento de coleta de fontes secundárias, referente a repercussões da sífilis na gestação e as possibilidades de atuação da enfermagem. A revisão de literatura é um tipo estudo que utiliza a análise e síntese do conhecimento científico já elaborado sobre a temática investigada, possibilitando obter informações para a elaboração da revisão (Botelho, de Almeida Cunha \& Macedo, 2011).

A coleta foi realizada em julho de 2020, no qual foram feitas buscas online por meio de palavras chaves préselecionadas, pesquisadas no banco de dados da Biblioteca Virtual de Saúde (BVS), e nas bases de dados Scientific Electronic Library Online (SCIELO), e U.S. National Library of Medicine (PubMed). 
Os Descritores em Ciências da Saúde (DeCS) foram combinados de diferentes formas para garantir uma busca ampla. Utilizou-se a combinação dos DeCS e o uso dos operadores booleanos AND e OR entre eles para delimitar a pesquisa: assistência de enfermagem OR sífilis AND saúde materno fetal OR gestação AND pré-natal.

Determinou-se como critérios de inclusão apenas artigos disponíveis na íntegra, gratuitos, publicados entre 2015 e 2020 em inglês, português ou espanhol. Os critérios de exclusão foram teses, dissertações, os duplicados e aqueles que não permitiam alcançar o objetivo traçado.

Encontrou-se na busca avançada da PubMed 11 artigos, Scielo 2, BVS 181, totalizando 194 artigos, sem os critérios de inclusão e exclusão, após a inserção destes, obteve-se um total de 86 artigos que foram analisados de forma sistemática por meio do tema e do resumo, excluindo aqueles que não abordavam o objetivo traçado e estudos duplicados; os artigos que restaram foram lidos e avaliados com mais rigor e foram excluídos aqueles que não respondiam à pergunta norteadora. Desse modo, foram elegíveis para compor esta revisão integrativa, somente 3 artigos, pois somente esses tinham informações mais completas a respeito do tema, como mostra a Figura 1.

Figura 1- Fluxograma de seleção dos estudos para construção desta revisão. Brasil, 2021.

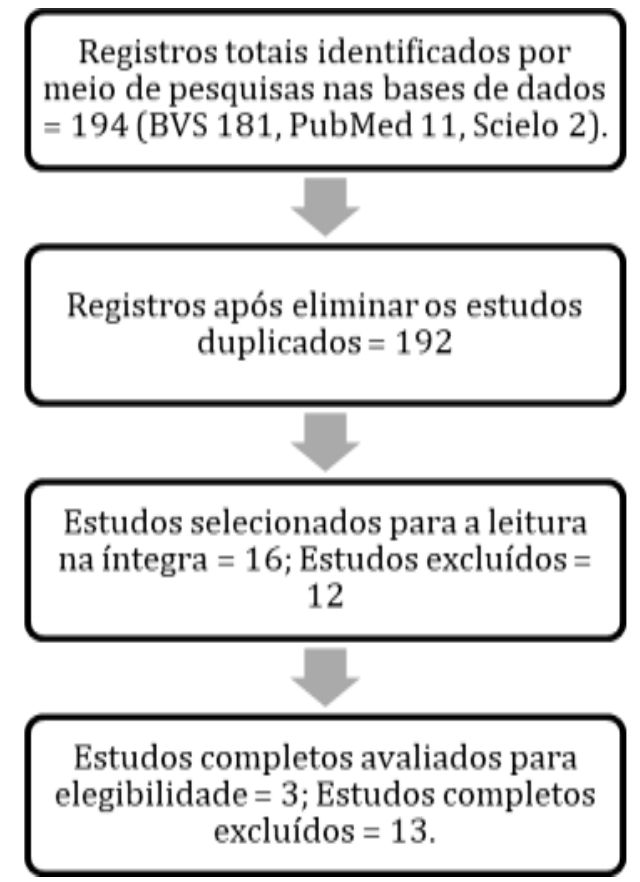

Fonte: Autores (2021).

\section{Resultados}

Foram averiguados 16 artigos de acordo com os critérios de elegibilidade, destes 12 apresentavam-se como indicativos para os critérios de exclusão, por não apresentarem especificidade com o tema e descreverem a sífilis gestacional sem abordar as repercussões materno fetal e as possibilidades de atuação do enfermeiro.

Para a busca dos artigos utilizamos os descritores de diversas formas para garantir uma busca ampla, utilizando operadores booleanos combinados. Conforme o Quadro 1 foram obtidos no total 194 artigos. Sendo que apenas 86 artigos se encaixavam nos critérios de inclusão e 16 foram selecionados através da leitura do título e do resumo. Para análise final foram selecionados 3 artigos pois respondiam a questão norteadora do estudo em questão. 
Quadro 1- Descrição das estratégias de busca, bases de dados, quantidade, título, resumo e textos na íntegra dos artigos analisados.

\begin{tabular}{|c|c|c|c|c|c|}
\hline $\begin{array}{c}\text { ESTRATÉGIA } \\
\text { DE BUSCA }\end{array}$ & $\begin{array}{c}\text { BASES DE } \\
\text { DADOS }\end{array}$ & QUANTIDADE & TÍTULOS & RESUMOS & SELECIONADOS \\
\hline $\begin{array}{c}\text { Syphilis and Nursing Care and } \\
\text { Pregnancy }\end{array}$ & PUBMED & 11 & 11 & 2 & 0 \\
\hline $\begin{array}{c}\text { Sífilis AND assistência de } \\
\text { enfermagem AND gestação }\end{array}$ & SCIELO & 2 & 1 & 1 & 0 \\
\hline $\begin{array}{c}\text { assistência de enfermagem OR } \\
\text { sífilis AND saúde materno fetal } \\
\text { OR gestação AND pré-natal }\end{array}$ & BVS & 181 & 74 & 13 & 3 \\
\hline
\end{tabular}

Fonte: Autores (2021).

Após as buscas, foram selecionados 3 artigos. Foi confeccionado um novo quadro contendo o título do artigo, autores, ano de publicação, país, periódico, método e resultados encontrados.

Quadro 2- Descrição dos artigos incluídos, segundo título, autores, ano de publicação, delineamento e resultados encontrados.

\begin{tabular}{|c|c|c|c|c|c|}
\hline Título & Autor/ano & País & Periódico & Método & Principais resultados \\
\hline $\begin{array}{lr}\text { Sífilis na gestação: } \\
\text { perspectivas } \\
\text { condutas } \\
\text { enfermeiro }\end{array}$ & $\begin{array}{l}\text { Targino Nunes, } \\
\text { Viana Marinho, } \\
\text { Barbosa Davim, } \\
\text { Gonçalo de Oliveira } \\
\text { Silva, Saraiva Felix } \\
\text { \& Figueiredo de } \\
\text { Martino (2017) }\end{array}$ & Brasil & $\begin{array}{l}\text { Rev. } \\
\text { enfermagem } \\
\text { UFPE } \\
\text { on line }\end{array}$ & $\begin{array}{l}\text { Estudo } \\
\text { qualitativo, tipo } \\
\text { descritivo } \\
\text { exploratório. }\end{array}$ & $\begin{array}{l}\text { Atuação do enfermeiro junto à } \\
\text { gestante com sífilis, seu papel como } \\
\text { educador de saúde, na promoção, } \\
\text { prevenção e tratamento da } \\
\text { enfermidade, evitando possíveis } \\
\text { complicações na saúde materno } \\
\text { fetal. Importância da notificação } \\
\text { compulsória e participação da } \\
\text { enfermagem neste processo. }\end{array}$ \\
\hline $\begin{array}{l}\text { Práticas de } \\
\text { enfermagem acerca do } \\
\text { controle da sífilis } \\
\text { congênita }\end{array}$ & $\begin{array}{l}\text { Beck \& Souza } \\
(2018)\end{array}$ & Brasil & $\begin{array}{l}\text { Revista Online } \\
\text { de Pesquisa }\end{array}$ & $\begin{array}{l}\text { Revisão narrativa } \\
\text { de literatura }\end{array}$ & $\begin{array}{l}\text { Práticas de enfermagem em ações } \\
\text { educativas, na investigação, } \\
\text { diagnóstico } \\
\text { precoce, } \\
\text { estabelecimento do tratamento. O } \\
\text { acolhimento dos parceiros na adesão } \\
\text { ao tratamento, para favorecer a } \\
\text { quebra da cadeia de transmissão, } \\
\text { avaliando as condições de risco para } \\
\text { saúde materno infantil, dessa forma, } \\
\text { controlando a SC. }\end{array}$ \\
\hline
\end{tabular}




\begin{tabular}{|l|l|l|l|l|l|}
\hline $\begin{array}{l}\text { Sífilis congênita no } \\
\text { recém-nascido: } \\
\text { repercussões para a } \\
\text { mãe }\end{array}$ & $\begin{array}{l}\text { da Silva, Gomes, } \\
\text { Ribeiro, Nobre, de } \\
\text { Oliveira Nörberg \& } \\
\text { Mota (2019). }\end{array}$ & Brasil & $\begin{array}{l}\text { Rev. enferm. } \\
\text { UERJ }\end{array}$ & Estudo qualitativo & $\begin{array}{l}\text { Repercussões do diagnóstico de SC } \\
\text { no }\end{array}$ \\
RN para a genitora, \\
predominando sentimentos de culpa, \\
desespero, tristeza, horror, medo do \\
estigma e falta de informação sobre \\
enfermidade e as possíveis \\
sequelas.
\end{tabular}

Fonte: Autores (2021).

\section{Discussão}

Tendo em vista os estudos dos artigos selecionados nesta revisão integrativa, obteve-se algumas informações para melhor sintetizar a temática. Delimitou-se em três categorias, são elas: repercussões da sífilis na mãe; consequências da transmissão vertical para o feto e/ou neonato; possibilidades de atuação da enfermagem.

O artigo de da Silva, Gomes, Ribeiro, Nobre, de Oliveira Nörberg \& Mota (2019), é um estudo qualitativo que aborda as repercussões do diagnóstico da SC no recém nascido para a mãe, evidenciando a falta de informações entre algumas gestantes sobre a possibilidade de transmissão vertical, a necessidade de internação hospitalar para o RN após o parto, e a importância do tratamento adequado para que não ocorra infecção, resultando em sentimentos negativos para a mãe (Silva et al., 2019). Dessa forma, demonstrou-se o quão essencial é o papel do enfermeiro no acolhimento e na orientação a mãe, promovendo a educação permanente em saúde.

Segundo Lima, Mororó, Feijão, Frota, Martins, \& Ribeiro (2016), as genitoras quando são informadas do diagnóstico de SC em seu filho, manifestam sentimento de tristeza, culpa e medo das complicações da doença. Apresentam também insegurança, fragilidades e falta de conhecimento em relação a enfermidade (Lima, Mororó, Feijão, Frota, Martins, \& Ribeiro, 2016). Nesse sentido, ainda que o diagnóstico de sífilis na mãe seja confirmado durante o pré-natal, algumas não realizam adequadamente o tratamento ou se infectam, resultando a um quadro de SC ao neonato (Lopes, de Araújo, Vasconcelos, Uchoa, Rocha \& dos Santos, 2016; da Silva, Gomes, Ribeiro, Nobre, de Oliveira Norberg \& Mota, 2019). A limitada compreensão em relação a doença, acrescentado a baixa escolaridade favorece a resistência a internação do RN após o parto, e neste momento é o que causa impacto em algumas delas ao perceberas consequências da SC e que o filho precisará de tratamento.

Lima et al. (2016), por meio de sua pesquisa, retratou os sentimentos das genitoras frente ao diagnóstico e ao tratamento da SC no nuelo, predominando culpa, desespero, tristeza e horror (LIMA et al, 2016). Com relação a reincidência da SC em mais de uma gestação, constataram que o impacto maior é no primeiro diagnóstico, depois da experiência ficam mais preparadas para enfrentar o posterior diagnóstico do RN. Outro ponto percebido foi o medo do estigma que envolve a doença, demonstrando preocupação em outras pessoas saberem da causa da hospitalização, o que leva a ocultação do motivo de internação aos familiares menos próximos.

A sífilis leva a sequelas a curto e longo prazo se não tiver o tratamento adequado para frear suas repercussões. Para a genitora as consequências vão desde físicas à sofrimento psíquico, e durante a gestação, quando ocorre a transmissão vertical, pode ocasionar abortamento, baixo peso ao nascer, prematuridade, natimorto e óbitos perinatais (Domingues \& Leal, 2016).

A sífilis congênita é classificada em precoce, diagnosticada antes de 2 anos de vida, e tardia após este período. Nesta perspectiva, a SC precoce pode apresentar características clínicas como: hepatomegalia, esplenomegalia, lesões cutâneas, periostite ou osteocondrite, pseudoparalisia dos membros, lesões no aparelho respiratório, convulsões, meningite, síndrome 
nefrótica, entre outros. Na SC tardia apresenta como principais características: dentes de Hutchinson, articulações de Clutton, fronte olímpica, nariz em sela, mandíbula curta, arco palatino elevado, surdez, dificuldade no aprendizado, ceratite intersticial (BRASIL, 2012).

Diante disto, é fundamental que se tenha o devido acompanhamento da gestante durante todo o desenvolvimento do seu período gestacional. Para isso, em 2000 foi instituído pelo Ministério da Saúde, o Programa de Humanização no Pré Natal e Nascimento (PHPN), com o objetivo de reduzir as possíveis repercussões negativas para a mãe e para o feto. Com isso, houve o aprimoramento e inclusão de questões como prevenção e detecção precoce da sífilis e posteriormente um tratamento mais adequado para este acometimento patológico, fazendo com que haja também uma preparação preventiva frente a transmissão vertical (Suto, Silva, Almeida, Costa \& Evangelista, 2016).

Targino Nunes, Viana Marinho, Barbosa Davim, Gonçalo de Oliveira Silva, Saraiva Felix \& Figueiredo de Martino (2017), retrata em seu artigo sobre a suma importância de realizar consultas pré-natais de qualidade, pois desta forma faz-se deste método como a primeira ferramenta de combate às taxas de morbimortalidade materno e infantil, principalmente em casos de mães portadoras de sífilis. Diante disto, a enfermagem tem uma importante participação neste momento crucial que é o pré-natal, tendo em vista que durante os atendimentos o(a) enfermeiro(a) pode detectar prováveis fatores de risco e com isso evitar possíveis complicações futuras para a genitora e para o neonato.

Os profissionais de enfermagem, assim como todos os outros na área da saúde envolvidos neste contexto, devem incluir nas suas atividades ações educativas acerca do tema, abordando as gestantes de forma inteligente e as estimulando a procurar ajuda e desta forma, conseguindo captar o maior número possível de genitoras portadoras de sífilis, e consequentemente iniciando o mais precocemente possível o tratamento das mesmas. Outro fator importante é o acolhimento dos parceiros e atraí-los também para a investigação da possibilidade de ele também ser um portador, e se positivo, iniciar medidas terapêuticas com ele, tendo em vista que a reinfecção da mulher perpetua a sífilis, o que pode fazer do tratamento dela ineficaz e implicar em consequências não só para ela como também para o feto (Beck \& Souza, 2018).

A sífilis é uma enfermidade de notificação compulsória, e a enfermagem tem papel importante na notificação dos casos, necessitando de qualificação adequada para que seja evitado a subnotificação (BRASIL, 2017). O sub registro é uma realidade do país que precisa ser revista, pois acarreta falhas nas políticas públicas de saúde, favorecendo a constante prevalência da sífilis em gestantes e SC no país (Favero, Ribas, Dalla Costa \& Bonafe, 2019). Portanto cabe ao profissional realizar a notificação, investigação e o tratamento com a prescrição e administração da penicilina, assim, resultando em uma assistência qualificada e na prevenção da transmissão vertical, possibilitando a análise de novas medidas para erradicar a doença.

\section{Considerações Finais}

$\mathrm{O}$ artigo retrata sobre as repercussões da sífilis na gestação e as possibilidades de atuação da enfermagem, onde foi acordado sobre a importância da(o) enfermeira(o) em ofertar um atendimento amplo e de qualidade perante as gestantes portadoras de sífilis, dando ênfase à atenção primária nas consultas de pré natal.

É perceptível que o Treponema pallidum desencadeia diferentes reações clínicas e psíquicas em cada gestante, interferindo na evolução natural do período gestacional, trazendo medo e angústia ao saberem das possíveis complicações que este quadro infeccioso pode acarretar tanto para ela, quanto para o feto. Com relação a criança que está sendo gerada, as repercussões variam, podendo causar diversas consequências negativas, dentre elas: prematuridade, baixo peso ao nascer e até mesmo óbito fetal. A mãe, ao saber da proporção que este quadro patológico pode chegar, demonstram sentimento de culpa, e quando o 
neonato nasce apresentando alguma comorbidade, surge a sensação de vergonha por aquilo ter ocorrido, e muitas vezes preferem manter a situação em sigilo.

Por isso, é de suma importância que a enfermagem atue de forma completa no atendimento prestado a genitora, tanto medicamentoso como educacional, lhes informando da importância de iniciar a terapêutica o quanto antes para frear a evolução negativa que a sífilis causará durante a gestação, tirando todas as suas dúvidas e conversar sobre a participação do companheiro, se estiverem mantendo relacionamento durante o período de acompanhamento, nas consultas e também no tratamento, pois se o companheiro também for portador da bactéria, poderá causar uma reinfecção da gestante e invalidar toda terapêutica realizada com ela. Ainda, faz-se necessários novos estudos acerca da temática a fim de embasar o cuidado de enfermagem a gestantes com sífilis.

\section{Referências}

Andreucci, C. B., \& Cecatti, J. G. (2011). Desempenho de indicadores de processo do Programa de Humanização do Pré-natal e Nascimento no Brasil: uma revisão sistemática. Cadernos de Saúde Pública, 27(6), 1053-1064.

Araújo, G. T. D. (2015). Análise do perfil de encaminhamento da atenção primária para pré-natal de alto risco: desafios para o ensino de competências em saúde materno-infantil (Master's thesis, Universidade Federal do Rio Grande do Norte).

Beck, E. Q., \& Souza, M. H. T. (2018). Práticas de enfermagem acerca do controle da sífilis congênita. Revista de Pesquisa: Cuidado é Fundamental Online, 10(Especial), 19-24.

Botelho, L. L. R., de Almeida Cunha, C. C., \& Macedo, M. (2011). O método da revisão integrativa nos estudos organizacionais. Gestão e sociedade, 5(11), 121-136.

Brasil (2012). Atenção ao pré-natal de baixo risco. Cadernos de Atenção Básica, $n$. 32.

Brasil. (2015). Protocolo clínico e diretrizes terapêuticas para atenção integral às pessoas com infecções sexualmente transmissíveis. http://www.aids.gov.br/pt-br/pub/2015/protocolo-clinico-e-diretrizes-terapeuticas-para-atencao-integral-pessoas-com-infeccoes

Brasil. (2017). Boletim Epidemiológico- Sífilis. http://www.aids.gov.br/pt-br/pub/2017/boletim-epidemiologico-de-sifilis-2017

Damasceno, A. B., Monteiro, D. L., Rodrigues, L. B., Barmpas, D. B. S., Cerqueira, L. R., \& Trajano, A. J. (2014). Sífilis na gravidez. Revista Hospital Universitário Pedro Ernesto, 13(3).

de Figueiredo, M. S. N., Cavalcante, E. G. R., de Oliveira, C. J., Monteiro, M. D. F. V., da Silva Quirino, G., \& de Oliveira, D. R. (2015). Percepção de enfermeiros sobre a adesão ao tratamento dos parceiros de gestantes com sífilis. Rev Rene, 16(3), 345-354.

Domingues, R. M. S. M., \& Leal, M. D. C. (2016). Incidence of congenital syphilis and factors associated with vertical transmission: data from the Birth in Brazil study. Cadernos de saude publica, 32(6).

Duarte, S. J. H., Borges, A. P., \& de Arruda, G. L. (2011). Ações de enfermagem na educação em saúde no pré-natal: relato de experiência de um projeto de extensão da Universidade Federal do Mato Grosso. Revista de Enfermagem do Centro-Oeste Mineiro.

Favero, M. L. D. C., Ribas, K. A. W., Dalla Costa, M. C., \& Bonafe, S. M. (2019). Sífilis congênita e gestacional: notificação e assistência pré-natal. Arquivos de Ciências da Saúde, 26(1), 2-8.

Guerra, H. S., da Costa, C. V., dos Santos, I. A. B., da Silva, J. M., \& Barcelos, T. F. (2017). Sífilis congênita: repercussões e desafios. Arquivos catarinenses de medicina, 46(3), 194-202.

Lima, V. C., Mororó, R. M., Feijão, D. M., Frota, M. V. V., Martins, M. A., \& Ribeiro, S. M. (2016). Mother's perception of congenital syphilis in her fetus. Revista de Saúde Pública do Paraná [Online], 17(2), 118-25.

Lopes, ACMU, de Araújo, MAL, Vasconcelos, LDPG, Uchoa, FSV, Rocha, HP, \& dos Santos, JR (2016). Implementação de testes rápidos para sífilis e HIV na assistência pré-natal em Fortaleza - Ceará. Revista Brasileira de enfermagem , 69 (1), 54-58.

Magalhães, D. M. D. S., Kawaguchi, I. A. L., Dias, A., \& Paranhos Calderon, I. D. M. (2011). A sífilis na gestação e sua influência na morbimortalidade materno-infantil. Comun. ciênc. saúde, 43-54.

Silva, J. G., Gomes, G. C., Ribeiro, J. P., Nobre, C. M. G., de Oliveira Norberg, P. K., \& Mota, M. S. (2019). Congenital syphilis in newborns: repercussions for the mother/Sifilis congenita no recem-nascido: repercussoes para a mae/Sifilis congenita en el neonato: repercusiones para la madre. Enfermagem Uerj, 27, NA-NA.

Silva, J. G., Gomes, G. C., Ribeiro, J. P., Nobre, C. M. G., de Oliveira Nörberg, P. K., \& Mota, M. S. (2019). Sífilis congênita no recém-nascido: repercussões para a mãe [Congenital syphilis in newborns: repercussions for the mother][Sífilis congénita en el neonato: repercusiones para la madre]. Revista Enfermagem UERJ, 27, 41031. 
Research, Society and Development, v. 10, n. 7, e51710716944, 2021

(CC BY 4.0) | ISSN 2525-3409 | DOI: http://dx.doi.org/10.33448/rsd-v10i7.16944

Suto, C. S. S., Silva, D. L. D., Almeida, E. D. S. D., Costa, L. E. L., \& Evangelista, T. J. (2016). Assistência pré-natal a gestante com diagnóstico de sífilis. Rev. enferm. atenção saúde, 18-33.

Targino Nunes, J., Viana Marinho, AC, Barbosa Davim, RM, Gonçalo de Oliveira Silva, G., Saraiva Felix, R., \& Figueiredo de Martino, MM (2017). sífilis na gestação: perspectivas e conduta do enfermeiro. Revista de Enfermagem UFPE / Revista de Enfermagem UFPE, 11 (12). 